\title{
Anomalia anorretal e cuidados maternos
}

\author{
Anorectal anomaly and maternal care \\ Anormalidades anorectales y la atención materna
}

\begin{abstract}
Manuela Costa Melo', Ivone Kamada'
'Universidade de Brasília. Escola Superior em Ciências da Saúde. Departamento de Enfermagem. Brasília, DF
\end{abstract}

Submissão: 04/08/2009

Aprovação: 12/09/2010

\section{RESUMO}

O objetivo deste artigo foi realizar um estudo de revisão bibliográfica sobre anomalia anorretal e cuidados maternos. O assunto foi abordado por meio de revisão integrativa realizada em consulta de artigos publicados nas bases de dados indexados na Biblioteca Virtual em Saúde. Foram identificadas 25 publicações que atenderam aos critérios de inclusão e exclusão pré-estabelecidos. Nos estudos analisados observaram-se distintos desenhos metodológicos demonstrando Que algumas crianças nascidas com anomalia anorretal necessitam de cirurgia de urgência. Os profissionais de saúde, juntamente aos pais, precisam desenvolver parcerias Que possibilitem um acompanhamento a longo prazo e orientações cuidadosas. Faz-se necessário Que novas pesquisas sejam realizadas sobre o tema, com propostas metodológicas que retratem melhor a essência do cuidado com as crianças estomizadas.

Descritores: Estomia; Cuidado do lactente; Enfermagem.

\section{ABSTRACT}

This purpose of the article was to make a bibliographic review about anorectal malformations and maternal cares. The matter was addressed through an integrative review undertaken in consultation of articles published in the databases indexed in the Virtual Health Library. We identified 25 publications that met the inclusion and exclusion criteria pre-established. In the studies reviewed, there were different study designs, demonstrating that some children born with anorectal anomalies reQuiring urgent surgery. Health professionals, along with the parents, need to develop partnerships that would enable a long-term monitoring and careful guidance. It is necessary to do more research on the subject, with methodological proposals that reflect the essence of the best care of an ostomized child.

Key words: Ostomy; Infant care; Nursing

\section{RESUMEN}

El objetivo de este artículo fue realizar un estudio de revisión bibliográfica sobre anomalía ano retal y cuidados maternos. El asunto fue abordado por medio de una revisión integral realizada por consulta de artículos publicados en las bases de datos indexados en la Biblioteca Virtual en Salud. Fueron identificadas 25 publicaciones Que atendieron a los criterios de inclusión y exclusión pre-establecidos. En los estudios analizados se observaron distintos dibujos metodológicos demostrando Que algunos niños nacidos con anomalía ano retal necesitan de cirugía de urgencia. Los profesionales de salud, juntamente con los padres, necesitan desarrollar grupos Que posibiliten un acompañamiento a largo plazo y orientaciones cuidadosas. Se hace necesario Que nuevos estudios sean realizados sobre el tema, con propuestas metodológicas Que retraten mejor la esencia del cuidado con los niños con ostomía.

Descriptores: Ostomía; Cuidado con el lactante; Enfermería. 


\section{INTRODUÇÃO}

A criança e a mãe possuem interações físicas, emocionais, psíquicas e sociais uma com a outra. É neste ambiente Que a criança interage Quase Que, exclusivamente, nos primeiros anos de sua vida, adQuirindo sua identidade, sua posição individual na rede de interações sociais. Portanto, o bebê ao nascer já pertence a uma rede familiar, continuando a estabelecer vínculos Que se iniciaram a partir do ventre materno. É sabido Que a criança, sendo um ser em crescimento e desenvolvimento, necessita de um ambiente de acolhimento e afeto para Que a atenção básica de cuidado, aprendizagem e carinho sejam atendidos. Na internação da criança é importante considerar os aspectos emocionais em relação ao seu desenvolvimento biopsicossocial. O profissional de saúde que dará a assistência procurará a compreensão do relacionamento mãefilho, apoiando, protegendo e fortalecendo, uma vez Que a sua presença afluirá para a efetivação do clima emocional desejável ${ }^{(1-2)}$.

Várias são as crianças nascidas com alguma malformação congênita e Que, muitas vezes, precisarão de internação para a resolução do problema. As malformações congênitas incluem as anomalias presentes no momento do nascimento, as morfológicas (malformações, deformidades) ou funcionais (retardo mental, cegueira, surdez). Sua etiologia pode ser genética ou ambiental e na maioria dos casos existe uma multifatoridade Que se interrelaciona; são os fatores genéticos predisponentes e fatores ambientais desencadeantes ${ }^{(3-4)}$. Uma das anomalias congênitas Que podem ocorrer é o nascimento da criança com ânus imperfurado e, por isso, em muitos casos, há a necessidade da realização de estomias.

As crianças necessitam fazer estomias por vários problemas de saúde, não apenas por nascer com ânus imperfurado, e esses estomas ocorrem de forma temporária ou definitiva, a reconstrução do trânsito, seja gastrintestinal ou urinário, depende da doença de base e das intervenções cirúrgicas necessárias. As causas mais freQüentes no lactente são enterocolite necrosante, ânus imperfurado, e, menos comum, doença de Hirschsprung; nas crianças maiores são as doenças intestinais inflamatórias, defeitos congênitos e as ureterostomias nos defeitos de bexiga ou da porção distal do uretér ${ }^{(2)}$.

No intuito de identificar os trabalhos alcançados a respeito desse tema, surgiu o interesse em realizar a revisão integrativa (RI). Foi determinado como objetivo principal para esta revisão, a identificação de pesquisas Que abordem as crianças com anomalia anorretal e cuidados maternos.

\section{MÉTODOS}

Tratou-se de uma RI a respeito do tema de crianças nascidas com anomalia anorretal e o cuidado materno. Essa revisão foi feita por leituras exploratória e seletiva do material de peseuisa indexados nos bancos de dados acessados eletronicamente na Biblioteca Virtual em Saúde (BVS) da Biblioteca Regional de Medicina (BIREME).

A RI é a revisão dos estudos disponíveis sobre um tópico em particular, e permite o agrupamento das evidências na prática clínica. Como toda revisão, esse método também possui a finalidade de reunir resultados, analisá-los de maneira sistemática e extrair a conclusão, com isso contribuir para o aprofundamento do conhecimento do tema investigado. Aponta e discute soluções para problemas similares e oferece alternativa de metodologias Que têm sido utilizadas para solução dos problemas. A RI vai além de uma revisão de literatura, devido o seu resultado possuir contribuições essenciais da literatura e conhecimentos específicos.

O caminho a ser percorrido para a realização de uma eficiente RI segue alguns pontos, como, primeiramente a identificação do tema, Que deve ser feita de forma clara e específica, com a formulação de uma hipótese Que apresente relevância para a saúde. Ao ser definido o assunto, segue-se para o ponto seguinte, encontrar os artigos científicos, a pesquisa é exaustiva nos bancos de dados, e para a seleção dos estudos faz-se necessário os critérios de inclusão e exclusão de artigos, caso contrário, ocorreria a inserção de todos os encontrados ${ }^{(5-6)}$. É importante que todas as decisões tomadas frente a esses critérios sejam documentadas e justificadas na descrição da metodologia da revisão.

O terceiro ponto possui como objetivo organizar as informações colhidas. O seguinte é a análise das informações colhidas, Que deverá ser realizada de forma crítica, e procurando responder a alguns Questionamentos, como: Qual é a Questão da pesQuisa; Qual é a base para esta pesquisa; por Que esta Questão é importante; como eram as Questões de pesquisas já realizadas; a metodologia do estudo está adequada; os sujeitos selecionados para o estudo estão corretos; o que a Questão da pesquisa responde; a resposta está correta e Quais pesQuisas futuras serão necessárias. O Quinto ponto corresponde a avaliação sistemática dos estudos incluídos, apontando sugestões para futuras pesQuisas direcionadas para a melhoria da assistência à saúde. Por último, a elaboração do documento Que deve observar a descrição das etapas percorridas e os principais resultados evidenciados na análise dos artigos incluídos ${ }^{(5-6)}$

A coleta de dados foi realizada no segundo semestre de 2006, utilizando a via eletrônica consultando as bases de dados. Compreendeu o período de publicação dos artigos entre os anos de 2002 e 2006, este período foi determinado em decorrência do início da coleta informações para o desenvolvimento do anteprojeto para a seleção no Programa de Pós-Graduação em Ciências da Saúde, no Curso para o Mestrado na Universidade de Brasília (UnB). Foram utilizados os seguintes descritores: relação mãe e filho; malformação anorretal.

A revisão iniciou-se com o levantamento bibliográfico, em seguida a realização de leitura exploratória do material encontrado, determinado pelo critério de inclusão e exclusão: pesquisas Que abordem os descritores estabelecidos, publicados na íntegra, entre os anos de 2002 e 2006; nos idiomas Português, Inglês, e Espanhol; e indexados nas bases de dados da Literatura Latino-Americana e do Caribe em Ciências da Saúde (LILACS), da Literatura Internacional em Ciências da Saúde (MEDLINE) e da Scientific Electronic Library Online (SciELO).

Em seguida, efetuou-se a leitura seletiva, a Qual permitiu determinar Qual material bibliográfico de interesse para a revisão. Em um segundo momento, a leitura na integra dos artigos com avaliação sistemática, e ao final, uma análise crítica para a elaboração dos temas. Foram identificados 376 artigos e, após a verificação dos critérios para a seleção do artigo e duplicidade, foram identificados 25, sendo encontrados 20 na base de dados da MEDLINE, três na LILACS e dois na SciELO.

$\mathrm{Na}$ avaliação sistemática observou-se Que as publicações apresentaram desenho e método distintos: dois utilizaram a 
observação participante e entrevista com os sujeitos; três o Questionário; um o interacionismo simbólico; Quatro o estudo de caso; três a revisão de literatura; e 12 o estudo retrospectivo. Totalizando-se 10 estudos Qualitativos e 15 Quantitativos, sendo Quatro no Idioma Português e 21 na Língua Inglesa.

Para melhor elaboração do documento final, foi realizada a análise crítica dos artigos escolhidos e organizados em dois temas: as conseqüências do nascimento de criança com anomalia anorretal e a família, a criança e o cuidado com a estomia. Os temas foram trabalhados de acordo com os artigos selecionados, e foram explorados da seguinte forma: 22 artigos encaixaram-se no desenvolvimento do primeiro tema e Quatro para o segundo.

\section{RESULTADOS E DISCUSSÂO}

\section{Consequências do nascimento de criança com anomalia anorretal}

Foi observado Que vários são os problemas neonatais Que necessitam de cirurgia de urgência, a anomalia anorretal é uma delas. O impacto do nascimento de uma criança mal formada é profundo, aliado ao problema da imperfuração anal inclui, aos pais e familiares, o desapontamento, a vergonha, os complexos de culpa, acusações mútuas, rejeições, preocupações financeiras e o medo das futuras gestações ${ }^{(3-4,7)}$.

A imperfuração anal é uma das anomalias anorretais e está associadas aos defeitos em outros sistemas do corpo $^{(3,8)}$, e estudos mostram a associação mais frequente com o trato urogenital ${ }^{(9-14)}$; a sobrevida depende da severidade das anomalias associadas. Logo no início deve-se obter uma anamnese sistemática e iniciar o tratamento, cirúrgico ou clínico, o mais precoce possível, a lesão se desenvolve devido à falta de complementação do complexo embriológico ${ }^{(15-19)}$.

O tratamento cirúrgico convencional é a confecção da colostomia pélvico proximal(20-21), em neonatos com um dia de vida, seguindo a formação da fístula com a realização da anorretoplastia sagital posterior, realizada aos 4 ou 6 meses de vida ${ }^{(22-23)}$. Após três meses dessa cirurgia é realizado o fechamento da colostomia, para isso é necessário ocorrer com frequência a dilatação suave da fístula anal no neonato, utilizando os dilatadores próprios ${ }^{(24)}$. Há chances de incontinência fecal em pacientes Que passaram por cirurgias em decorrência da malformação anorretal ${ }^{(25)}$, o tratamento do intestino à base de enemas, laxantes e medicamentos são bem sucedidos, Quando bem administrados.

Por isso, a importância da orientação à mãe, para não esperar, apenas, uma evolução normal do treinamento intestinal nessas crianças $^{(25)}$. Esse tipo de cirurgia, como Qualquer outra, deve ser vista no seu aspecto global. Informações simples relacionadas aos cuidados, incluindo a descrição da cirurgia, os procedimentos e o centro cirúrgico contribuirão para redução da angústia, do medo e da ansiedade por desconhecimento das situações.

\section{A família, a criança e o cuidado com a estomia}

A necessidade emocional mais importante para a criança é a de ser amada e a de sentir-se segura desse amor. Essa relação mãefilho é desenvolvida desde a vida intra-uterina e é fundamental o contato mãe e filho nos momentos iniciais da vida pós-natal. A família de uma criança hospitalizada passa por um período de adaptação, ocorrendo algumas transformações em relação aos aspectos psicológicos e comportamentais, pois a criança doente fica superprotegida, os irmãos expressam sentimentos de rejeição e abandono pelos pais e no próprio relacionamento dos pais ocorrem mudanças, essas transformações tanto podem fortalecer o casal, Quanto afastá-los, isso, dependerá da própria dinâmica familiar existente ${ }^{(1,26)}$.

Quando os pais são devidamente atendidos em suas necessidades, participam efetivamente nos cuidados do filho, beneficiam tanto a família Quanto a criança. Essa participação diminui o nível de ansiedade deles e da criança, contribuindo para melhor adaptação de ambos à situação hospitalar e na continuidade do tratamento após a alta. Para transformar a permanência fatigante em agradável dos familiares, na unidade, além do preparo dos profissionais de saúde para lidar com ela, é necessário Que família encontre seu modo de participar dos cuidados. Para isso, pode ser necessário Que a equipe organize seu processo de trabalho levando em conta as aptidões da família e suas necessidades de cuidado ${ }^{(27)}$.

A participação e colaboração dos familiares no processo de cuidado visam uma rápida recuperação e reabilitação do paciente, essas medidas, Quando adotadas, trazem benefícios e podem prevenir complicações. Desta forma a assistência aos pacientes estomizados deve ser praticada desde a fase pré, pós-operatória e alta hospitalar, a orientação emerge como uma estratégia Que integra a valorização e o reconhecimento da família no cuidado ao estomizado. Essa assistência envolve relacionamento interpessoal, constituído de atitudes humanas Que não podem ser preestabelecidas como uma função essencialmente técnica ${ }^{(27)}$.

Em relação à orientação no pré-operatório, desenvolve-se a aprendizagem da família, explicando como ocorrerá o procedimento, isso resultará em diminuição de complicações, redução do período de internação e diminuição da ansiedade ${ }^{(2,27)}$. Também está envolvido na assistência à pessoa estomizada a seleção do sistema de bolsa, tipos de barreira protetora e produtos acessórios a serem utilizados. Isso só se torna possível com o respaldo dos avanços tecnológicos alcançados pelos coletores específicos ao cuidado dos estomas e Que estão disponíveis no mercado.

A orientação, Que se inicia no pré-operatório tem continuidade no pós-cirúrgico e após a alta hospitalar. A atividade educativa do profissional de saúde é entendida como um meio para Que a família desenvolva suas potencialidades para o autocuidado. Sob essa perspectiva, ao enfermeiro cabe facilitar ou ajudar a se desenvolver para tal, a valorização da orientação para a alta refere-se ao desenvolvimento da independência e segurança ${ }^{(27)}$.

\section{CONSIDERAÇÕES FINAIS}

Foi percebida a necessidade do envolvimento da mãe e do pai nos cuidados integrais com o filho, na tentativa de possibilitar um acompanhamento a longo prazo e de orientações cuidadosas por meio dos profissionais de saúde, para não esperar, apenas, uma evolução normal do treinamento intestinal nos seus filhos. Para esse efetivo envolvimento, observou-se a falta de publicações Que descrevam projeto terapêutico de orientação para o cuidado, como também aos pais e outros familiares na assistência direta.

Demonstrou-se, ainda, Que a criança nascida com malformação anorretal requer uma avaliação cuidadosa, diagnóstico clínico correto, reconstrução anatômica meticulosa e compreensão nos 
cuidado pré e pós-operatório, com a finalidade da criança ter uma boa Qualidade de vida. Essas intervenções próprias possuem a finalidade de minimizar a morbidade .

Entende-se que este estudo tenha fornecido dados Que permitam reflexões para aprimorar a atuação dos familiares na atenção aos cuidados com a criança nascida com anomalia anorretal, esse é um desafio na busca da Qualidade para o cuidado. Porém, faz-se necessário Que novas pesquisas sejam realizadas, com propostas metodológicas Que retratem a essência do cuidado com as crianças estomizadas.

\section{REFERÊNCIAS}

1. Collet N, Rocha SM. Participação e autonomia da mãe no cuidado ao filho hospitalizado. Rev Bras Enferm 2003; 56(3): 260-4.

2. Barreire GS, Oliveira AO, Kazama W, Kimura M, Santos VLG. Qualidade de vida de crianças ostomizadas na ótica das crianças e das mães. I Pediatr 2003; 79(1): 55-61.

3. Ratan SK, Rattan KN, Pandey RM, Mittal A. Associated congenital anomalies in patients with anorectal malformations a need for developing a uniform practical approach. I Pediatr Surg $2004 ; 39$ (1 1): 1706- I I.

4. Goyal A, Williams IM, Kenny SE, Lwin R, et al. Functional outcome and Quality of life in anorectal malformations. I Pediatr Surg 2006; 4 I (2): 3 I 8-22.

5. Mendes KDS, Silveira RCCPS, Galvão MC. Revisão integrativa: método de pesquisa para a incorporação de evidências na saúde e na enfermagem. Rev Latino-am Enfermagem 2008; 17(4): 758-64.

6. Beyea SC, Nicoll LH. Writing an integrative review. AORN I 1998; 67(4): 877-80.

7. Kumar A, Agarwala S, Srinivas M, Bajpai M, et al. Anorectal malformations and their impact on survival. Indian I Pediatr 2005; 72(12): 1039-42.

8. Moënne BK. Imágenes en anomalias anorectales. Rev Chilena Radiol 2003; 9(1): 13-8.

9. Banu T, Hannan MJ, Aziz MA, Hoque M, et al. Rectovestibular fistula with vaginal malformations. Pediatr Surg Int 2006; 22(3): 263-6.

10. Hulthén MV, Mellstam L, Amark P, Frenckner B. Neurovesical dysfunction in children after surgery for high or intermediate anorectal malformations. Acta Pediatr 2004; 93(1): 43-6.

I1. Mosiello G, Capitanucci ML, Gatti C, Adorisio O. How to investigate neurovesical dysfunction in children with anorectal malformations. J Urol 2003; 170(4): 1610-3.

12. Rintala RJ. Fecal incontinence in anorectal malformations, neuropathy, and miscellaneous conditions. Semin Pediatr Surg 2002; I I (2): 75-82.

13. Saha SR, Roy AK; Saha S. Incidence of associated congenital anomalies in anorectal malformations. I Indian Med Assoc 2005; 103(12): 690-1.

14. Singal AK, Bhatnagar V, Agarwala S, Mitra DK.. Urethral duplication in association with anorectal malformation. Department of Paediatric Surgery, All India Institute of Medical Sciences, New
Delhi, India. Eur I Pediatr Surg 2006; 16(3): 1 14-6.

15. Levitt MA, Peña A. Outcomes from the correction of anorectal malformations. Curr Opin Pediatr 2005; 17(3): 394-401.

16. Sanjuan RS, González MM, Santamaría OJI. Perineal canal. Cir Pediatr 2003; 16(1): 48-50.

17. Holschneider A, Hutson I, Peña A, Beket E, et al. Preliminary report on the International Conference for the Development of Standards for the Treatment of Anorectal Malformations. J Pediatr Surg 2005; 40(10): 152 I-6.

18. Adejuyigbe O, Abubakar AM, Sowande AO, Olayinka OS, et al. Experience with anorectal malformations in Ile-Ife, Nigeria. Pediatr Surg Int 2004; 20(1 1-12): 855-8.

19. Pakarinen MP, Goyal A, Koivusalo A, Baillie C, et al. Functional outcome in correction of perineal fistula in boys with anoplasty versus posterior sagittal anorectoplasty. Pediatr Surg Int 2006; 22(12): 96I-5.

20. Archibong AE, Idika IM. Results of treatment in children with anorectal malformations in Calabar, Nigeria. S Afr I Surg 2004; 42(3): 88-90

21. Chalapathi G, Chowdhary SK, Rao KL, Samujh R. Risk factors in the primary management of anorectal malformations in Northern India. Pediatr Surg Int 2004; 20(6): 408- 11 .

22. Tsuji H, Okada A, Nakai H, Azuma T. Follow-up studies of anorectal malformations after posterior sagittal anorectoplasty. I Pediatr Surg 2002; 37(1): 1529-33.

23. Holschneider AM, Jesch NK, Stragholz E, Pfrommer W. Surgical methods for anorectal malformations from Rehbein to Peña critical assessment of score systems and proposal for a new classification. Eur I Pediatr Surg 2002; I 2(2): 73-82.

24. Peña A, Hong AR, Midulla P, Levitt M. Reoperative surgery for anorectal anomalies. Semin Pediatr Surg 2003; 12(2): 1 18-23.

25. Zuccarello B, Romeo C, Scalfari G, Impellizzeri P, et al. Scintigraphic evaluation of colonic motility in patients with anorectal malformations and constipation. I Pediatr Surg 2006; $41(2): 310-13$

26. Pinto IP, Ribeiro RC, Silva CV. Procurando manter o equilíbrio para atender suas demandas e cuidar da criança hospitalizada: a experiência da família. Rev Latino-am Enfermagem 2005; 6(13): 974-81.

27. Gemelli LMG, Zago MMF. A interpretação do cuidado com o ostomizado na visão do enfermeiro: um estudo de Caso. Rev Latino-am Enfermagem 2002; I (10): 34-40. 